This is the post peer-review accepted manuscript of:

M. P. Caro, M. S. Ali, M. Vecchio and R. Giaffreda, "Blockchain-based traceability in Agri-Food supply chain management: A practical implementation," 2018 loT Vertical and Topical Summit on Agriculture - Tuscany (IOT Tuscany), Tuscany, 2018, pp. 1-4.

The published version is available online at:

https://doi.org/10.1109/IOT-TUSCANY.2018.8373021

C2018 IEEE. Personal use of this material is permitted. Permission from IEEE must be obtained for all other uses, in any current or future media, including reprinting/republishing this material for advertising or promotional purposes, creating new collective works, for resale or redistribution to servers or lists, or reuse of any copyrighted component of this work in other works. 


\title{
Blockchain-based Traceability in Agri-Food Supply Chain Management: A Practical Implementation
}

\author{
Miguel Pincheira Caro*, Muhammad Salek Ali ${ }^{\dagger}$, Massimo Vecchio ${ }^{\ddagger}$ and Raffaele Giaffreda ${ }^{\S}$ \\ OpenIoT Research Area, FBK CREATE-NET, Italy \\ Email: *mpincheiracaro@fbk.eu, ${ }^{\dagger}$ ms.ali@fbk.eu, ${ }^{\ddagger}$ mvecchio@fbk.eu, ${ }^{\S}$ rgiaffreda@fbk.eu
}

\begin{abstract}
The recent, exponential rise in adoption of the most disparate Internet of Things (IoT) devices and technologies has reached also Agriculture and Food (Agri-Food) supply chains, drumming up substantial research and innovation interest towards developing reliable, auditable and transparent traceability systems. Current IoT-based traceability and provenance systems for Agri-Food supply chains are built on top of centralized infrastructures and this leaves room for unsolved issues and major concerns, including data integrity, tampering and single points of failure. Blockchains, the distributed ledger technology underpinning cryptocurrencies such as Bitcoin, represent a new and innovative technological approach to realizing decentralized trustless systems. Indeed, the inherent properties of this digital technology provide fault-tolerance, immutability, transparency and full traceability of the stored transaction records, as well as coherent digital representations of physical assets and autonomous transaction executions. This paper presents AgriBlockIoT, a fully decentralized, blockchain-based traceability solution for Agri-Food supply chain management, able to seamless integrate IoT devices producing and consuming digital data along the chain. To effectively assess AgriBlockIoT, first, we defined a classical use-case within the given vertical domain, namely from-farm-to-fork. Then, we developed and deployed such use-case, achieving traceability using two different blockchain implementations, namely Ethereum and Hyperledger Sawtooth. Finally, we evaluated and compared the performance of both the deployments, in terms of latency, CPU, and network usage, also highlighting their main pros and cons.
\end{abstract}

\section{INTRODUCTION}

Today, the vast majority of traditional logistic information systems in Agriculture and Food (Agri-Food) supply chains merely track and store orders and deliveries, without providing features as transparency, traceability and auditability. These features would surely improve food quality and safety, therefore they are more and more requested by consumers [1]. Thus, several Research \& Development communities are concentrating their efforts on adopting some specific Internet of Things (IoT) technologies such as RFIDs and Wireless Sensor Networks, or everyday-cheaper connected devices, to enabled remote monitoring of the conditions in food transportation scenarios and at a very fine granularity along the whole AgriFood supply chain, e.g., from production to consumption [2].

However, the majority of the current IoT solutions still rely on heavily-centralized cloud infrastructures, where there is usually a lack of transparency, and by nature presents security threats including availability, data lock-in, confidentiality and auditability [3].

In the Agri-Food domain, in order to maintain trust and reliability along the whole supply chain, it is essential for the stored records to be tamper-proof, while the best case would be if each actor issuing transactions could do that without relying on any centralized third-party intermediary. A potential solution to alleviate all of such issues and concerns is the Blockchain technology, which is a peer-to-peer digital ledger that does not rely on centralized servers. Since all the records stored in a blockchain are based on a consensus reached at least by the absolute majority of peers of the network itself, this distributed ledger is immutable by design and offers an auditable and transparent source of information. And from an IoT perspective, instead of requiring connectivity to a central cloud, sensor networks in a blockchain-based traceability solution would only require stable connection to their closely located peer. Thus, blockchains exposes all the required properties for decentralizing food traceability systems, while making traceable data available at every step of the supply chain.

In this paper, we present AgriBlockIoT, a fullydecentralized traceability system for the Agri-Food supply chain management. Specifically, the proposed solution can rely either on the Ethereum ${ }^{1}$ or the Hyperledger Sawtooth ${ }^{2}$ publicly available blockchain implementations, while it is able to integrate various IoT sensor devices. By directly producing and consuming valuable information from the IoT devices along the whole supply chain and storing such data directly in its underlying blockchain, AgriBlockIoT guarantees transparent and auditable asset traceability. To assess the feasibility of the proposed solution, we engineered and deployed the socalled from-farm-to-fork use-case: a classical food traceability scenario fostering certified traceability of food along the whole supply chain, e.g., from agricultural production (the farmside) to consumption (the fork-side). Then, we compare the two implementations, in terms of three performance metrics, namely latency, CPU load, and network usage.

The remaining of this paper is organized as follows: Sec. II summarizes the current state of the art in the adoption of blockchains as an enabling technology for the traceability in Agri-Food supply chains; Sec. III describes the system architecture of AgriBlockIoT; Sec. IV contains the analysis of our preliminary results; Sec. V concludes the paper.

\footnotetext{
${ }^{1}$ https://www.ethereum.org/

${ }^{2} \mathrm{https} / / / \mathrm{www}$.hyperledger.org/projects/sawtooth
} 


\section{RELATED WORK}

The last few years have witnessed an explosion of research and development activity around the Blockchain technology, mainly within the financial technology (FinTech) industry. Indeed, its intrinsic capability of providing immutable and tamper-proof records, together with its potential of enabling trust and reliability among untrusted peers represent too attractive features, preventing this technology to stay relegated into a single vertical sector. For this reason, several industries beyond the FinTech sector have already identified the Blockchain technology as a driver for a paradigm shift. For data reliability, ProvChain [4] explored the use of the Blockchain technology in a cloud storage scenario to verify three levels of data provenance: collection, storage and validation. In this work, the use of blockchains showed good results in terms of tamperproof records and user privacy, with very low overhead for the storage itself. In a similar context, the authors on [5] explored the use of blockchains with smart-contracts to achieve secure data provenance, using the Open Provenance Model (OPM) with an access control-based privacy-preserving solution.

Also the adoption of some IoT devices and technologies in the supply chain management sector has attracted a lot of research interest in the last few years. From the impact of autonomous identification system [6] to the application of RFID technologies in logistics [7], the technological maturity of the devices and of the sensors is literally revolutionizing each step of the process. Specifically for the Agri-Food domain, the authors of [8] presented an inventory transparency use-case, also adopting some IoT devices. There, the goal was to explore the use of RFID and NFC-based devices to achieve transparency and real-time information production directly on the field, enabling persistence by means of a centralized, cloud-based database. This is indeed the classical paradigm adopted by far the majority of the current IoT-based solutions.

However, the use of both the Blockchain and the IoT technologies in the Agri-Food domain is still an underexplored, yet worth-to-explore, research field. A traceability system based on the blockchain and the RFID technology was proposed in [9], with a sharp focus on Chinese food markets. The work considered fresh food asset tracking as fruits, vegetables and meat, by means of RFID-based devices for the data acquisition and blockchains for data persistence. The authors of [10] presented a supply chain traceability system for food safety, based on HACCP (Hazard Analysis and Critical Control Points) and focus on transparency. There, they described the process of crop plants in different phases, from harvesting to retailing, without going into the details of a performance analysis. Overall, to the best of our knowledge, some key-features offered by certain blockchain implementations remain either not explored, or not fully exploited, one for all being the autonomous transactions capability (often referred to as smart contracts [5]).

\section{AgriBlockIoT: System ARCHITECTURE}

The unique constrains and requirements of the modern Agri-Food industry pose some major challenges to achieve a transparent, auditable and reliable supply chain management process . Some of these challenges are the heterogeneity of the involved actors, stakeholders and business models, their different levels of confidentiality, the lack of interoperability among the involved systems and, most notably, the complete lack of a clear data governance [11]. Fig. 1 depicts a simplified version of such process, whose involved actors are briefly introduced in the following:

A) provider: providers of raw materials, such as seeds and nutrients, but also pesticides, chemicals, etc;

B) producer: usually the farmer e.g., the responsible of the actions from seeding/planting to harvesting;

C) processor: this actor may perform various actions, from simple packaging to more complex processes (e.g., pressing of the olives);

D) distributor: this actor is responsible of moving the output of the processor (e.g., the product) from processor's site to retailers;

E) retailer: this actor is responsible of selling the products, representing it either small local stores or big supermarkets;

F) consumer: the final element of the chain.

Along the whole process, authorities provide standards, regulations, laws, rules and policies that the involved actors have to comply with.

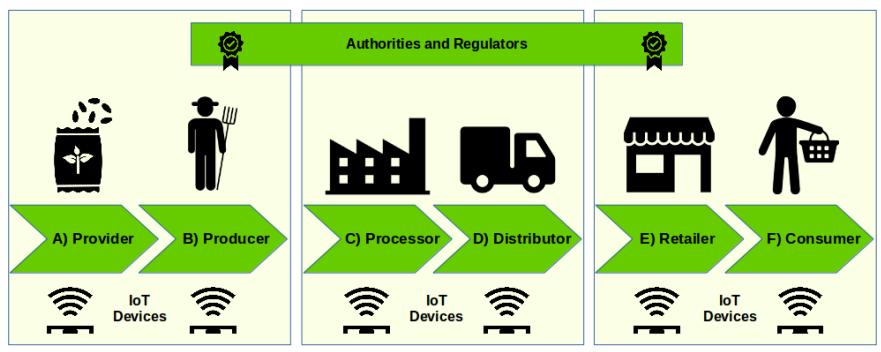

Fig. 1. Simplified version of the Agri-Food supply chain management process.

We propose a layered architecture able to rely on the Blockchain and the IoT technologies to achieve transparency, auditability and immutability of the stored records in a trustless environment. We consider the blockchain as a layer of our system (see Fig. 2), allowing AgriBlockIoT to be blockchainindependent, while it can be integrated into existing traditional software systems (ERP, CRM, etc.).

The proposed architecture takes advantage of the increasing capabilities offered by modern edge devices (e.g., gateways, mini-PC, etc.), which may be directly used as full nodes of our layered blockchain implementation, hence extending the resistance, decentralization, security and trust of the whole network. The main modules of AgriBlockIoT are :

- API: a REST Application Programming Interface exposing the capabilities of AgriBlockIoT to other applications, with a high level of abstraction, allowing easy integration with existing software systems;

- Controller: a component responsible of transforming the high-level function calls into the corresponding low-level 


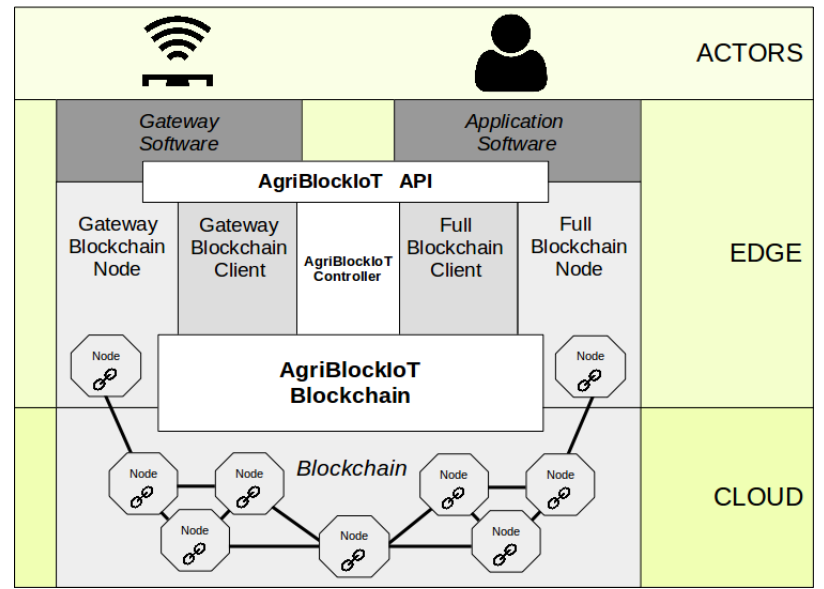

Fig. 2. Layered architecture of AgriBlockIoT.

calls for the blockchain layer, and viceversa (i.e., querying and converting the data records stored in the blockchain, into high-level information for the upper layer).

- Blockchain: The main component of the system, containing all the business logic, implemented through smart-contracts on the blockchain, as a gateway to the blockchain itself. Depending on the selected blockchain, this module will vary in complexity, according to the program capabilities of the selected blockchain, as well as the capabilities of the client interfaces for that blockchain.

Then, to coherently define the high-level functionality of AgriBlockIoT, we had a bottom-up approach through which we extracted the set of requirements starting from a complete use-case, namely from-farm-to-fork. The latter is, indeed, a classical food traceability use-case that fosters certified traceability of food along the whole supply chain, from agricultural production to consumption. In other words, AgriBlockIoT shall provide consumers with complete history of the food he is buying. The only pre-condition is that all the participants (so including the IoT devices) are registered users of the underlying blockchain, meaning that they have the correct public/private key-pairs to digitally sign each operation on the distributed ledger. In the following, we summarize the list of extracted requirements:

R1: Raw Materials Purchasing: producers and providers store in the blockchain the details of sales and purchases of raw materials, including technical information of products and amounts. Note: smart-tags (e.g., barcode, QR codes) can be used to automatize this process;

R2: Planting: producers store in the blockchain information about the planting process (e.g., the amount of seeds used). Note: sensors can automatize such data entry process (e.g., connected weight scales), while smart contracts can autonomously fire, hence creating records whenever anomalies are detected (e.g., more seeds than the ones registered as purchased);

R3: Growing: sensors, at regular intervals, autonomously store in the blockchain information about the grow- ing plants and environment. Note: smart contracts can asynchronously fire, hence creating records whenever anomalies are detected (e.g., sensor values outside certain thresholds);

R4: Farming: farmers store in the blockchain information about each stage of the process (e.g., irrigation, fertilizing, etc.), including amounts of inputs applied. Note: sensors can automatize such data entry process (e.g., chemical sensors and multisensory systems), while smart contracts can autonomously fire, hence creating records whenever anomalies are detected (e.g., sensor values outside certain thresholds);

R5: Harvesting: farmers store in the blockchain details about the harvesting. Note: sensors can automatize such data entry process (e.g., connected weight scales), while smart contracts can autonomously fire, hence certifying that the process from seeding to harvesting is compliant with certain regulations (e.g., organic, fair trade, etc.);

R6: Delivery to processor: farmers transfer the ownership of the products to distributors, directly through the blockchain. Note: sensors (e.g., GPS sensors) and smart contracts can automatize this process, or create records whenever anomalies are detected during the delivery phase (e.g., sensor values outside certain thresholds);

R7: Processing: considering the simplest case of a packaging processor, the latter store in the blockchain details about the received amount of product from distributors, the packaged amount and, eventually, the amount of product lost during the processing phase. Note: sensors can automatize such data entry process (e.g., connected weight scales), while smart contracts can autonomously fire, hence creating records whenever anomalies are detected (e.g., the packaged amount is larger than the received amount);

R8: Delivery to retailers: processors transfer the ownership of the processed product to distributors, directly through the blockchain. Note: sensors (e.g., GPS sensors) and smart contracts can automatize this process, or create records whenever anomalies are detected during the delivery phase (e.g., sensor values outside certain thresholds);

R9: Retailing: retailers store in the blockchain details about the received amount of product from distributors. Then, at regular intervals, sensors autonomously store in the blockchain information about the status of the retail environment. Note: smart contracts can asynchronously fire, hence creating records whenever anomalies are detected (e.g., sensor values outside certain thresholds);

R10: Consuming: retailers store in the blockchain details about the sold products, while consumers are able to transparently verify the whole history of a product before buying it. Note: smart-tags can be associated to each package, so that consumers can easily retrieve the whole history of the product. 


\section{Preliminary Results}

We assess the performance of AgriBlockIoT implementing the functionality of an IoT sensing device producing digital values that are directly stored in the blockchain. The stored data can be then retrieved, while it is possible to implement smart-contracts that are autonomously executed upon the occurrence of certain conditions on the data produced by the sensor itself. Since AgriBlockIoT is blockchain-agnostic, we implemented the underlying blockchain module over two different, private, six-nodes-based implementations, namely Ethereum and Hyperledger Sawtooth. The reasons of choosing these implementations are the different levels of customization for the records included on the ledger (transactions). While both platforms allow to implement complex business logic, Ethereum works with a single transaction structure, while Hyperledger Sawtooth allows the definition of a custom transaction structure.

Both the networks were configured with the default settings, and deployed in dedicated virtual machines equipped with 4GB of RAM, 2 Intel(R) Core(TM) i5-6440HQ CPUs $2.60 \mathrm{GHz}$ and $20 \mathrm{~GB}$ of hard disk. Regarding the Operating System, we opted for a fresh Linux Ubuntu 16-04 basic distribution, only installing the packages needed to deploy the corresponding blockchain node. A series of 100 test where run independently for each scenario. During each test, AgriBlockIoT simply set the value of a sensor, as done by an environmental IoT sensing device through a gateway, and issued a transaction in the blockchain. For each test we measured the time necessary to set the value in the blockchain (latency), the processing power of each node (CPU load), and the network usage (in terms of bytes transmitted and received); the average values are summarized in Table I. From these results, we observe that Hyperledger Sawtooth has better performances with respect to the Ethereum counterpart.

TABLE I

Performance of AgriBlockIoT IN TERMS OF LATENCY, NETWORK TRAFFIC, AND CPU LOAD.

\begin{tabular}{|lcccc|}
\hline & $\begin{array}{c}\text { latency } \\
\text { [seconds] }\end{array}$ & $\begin{array}{c}\text { network tx } \\
\text { [bytes] }\end{array}$ & $\begin{array}{c}\text { network rx } \\
\text { [bytes] }\end{array}$ & $\begin{array}{c}\text { CPU load } \\
{[\%]}\end{array}$ \\
\hline Ethereum & 16.55 & $528^{\prime} 108$ & $682^{\prime} 415$ & 46.78 \\
Sawtooth & 0.021 & $19^{\prime} 303$ & $20^{\prime} 641$ & 6.75 \\
\hline
\end{tabular}

\section{CONCLUSIONS}

AgriBlockIoT enables the integration of IoT and Blockchain technologies, creating transparent, fault-tolerance, immutable and auditable records which can be used for an Agri-Food traceability system. Regarding the preliminary, very practical test: even if the Hyperledger Sawtooth-based implementation had better results in terms of measured metrics with respect to the Ethereum one, both implementations have different properties and capabilities that need to be considered before choosing one over the other. In some cases it may be convenient to trade off the high-latency of Ethereum with its scalability and reliability, since it enables larger numbers of participants and its software maturity is far higher than Hyperledger Sawtooth. Moreover, from an economic perspective, recall that the monetary cost of using the Ethereum network can be avoided, simply by using private networks. However, in this environment, the limitation of having a single language for implementing smart-contracts, as well as a fixed structure for the records, may represent a drawback when developing more sophisticated business logic. Last but not least, the current consensus algorithm of Ethereum is quite CPU-intensive and this may represent a barrier for computationally-limited devices, such as edge gateways and IoT devices. On the other hand, the Hyperledger Sawtooth implementation offers a novel consensus algorithm which may be more suitable for such tiny devices. Furthermore, the ability of implementing the logic using different languages, as well as the customization of the records, may enable faster implementations and easier integrations with other systems. However, Hyperledger Sawtooth is still far for being considered a mature implementation at the level of Ethereum.

As future works, we plan to extend the performance analysis to more constrained hardware architectures, in order to assess the suitability of the proposed framework to applications comprising real IoT devices and gateways along the Agri-Food supply chain.

\section{ACKNOWLEDGMENT}

This work was supported by the AGILE project (grant agreement No 688088) within the Horizon 2020 programme of the European Union.

\section{REFERENCES}

[1] C. Verdouw, H. Sundmaeker, F. Meyer, J. Wolfert, and J. Verhoosel, "Smart agri-food logistics: requirements for the future internet," in Dynamics in Logistics. Springer, 2013, pp. 247-257.

[2] F. TongKe, "Smart agriculture based on cloud computing and IoT," Journal of Convergence Information Technology, vol. 8, no. 2, 2013.

[3] M. Armbrust, A. Fox, R. Griffith et al., "A view of cloud computing," Commun. ACM, vol. 53, no. 4, pp. 50-58, 2010.

[4] X. Liang, S. Shetty, D. Tosh, C. Kamhoua, K. Kwiat, and L. Njilla, "ProvChain: A blockchain-based data provenance architecture in cloud environment with enhanced privacy and availability," in Proc. of the CCGRID, 2017, pp. 468-477.

[5] A. Ramachandran and K. Murat, "Using blockchain and smart contracts for secure data provenance management," arXiv preprint arXiv:1709.10000, 2017.

[6] D. McFarlane and Y. Sheffi, "The impact of automatic identification on supply chain operations," The International Journal of Logistics Management, vol. 14, no. 1, pp. 1-17, 2003.

[7] C. Sun, "Application of RFID technology for logistics on Internet of Things," AASRI Procedia, vol. 1, pp. 106-111, 2012.

[8] S. Srinivasan, D. Shanthi, and A. Anand, "Inventory transparency for agricultural produce through IoT," IOP Conf. Ser: Mater. Sci. Eng, vol. 211, no. 1, p. 012009, 2017.

[9] F. Tian, "An agri-food supply chain traceability system for china based on rfid and blockchain technology," in Proc. of the ICSSSM. IEEE, 2016, pp. 1-6.

[10] F. Tian, "A supply chain traceability system for food safety based on HACCP, blockchain and Internet of Things," in Proc. of the ICSSSM, 2017, pp. 1-6.

[11] C. Brewster, I. Roussaki, N. Kalatzis, K. Doolin, and K. Ellis, "IoT in Agriculture: Designing a Europe-Wide Large-Scale Pilot," IEEE Commun. Mag., vol. 55, no. 9, pp. 26-33, 2017. 